\title{
Documental interactivo iberoamericano: proximidad y transformación social
}

\author{
Jorge Vázquez-Herrero \& Gisela Moreno*
}

Resumo: Este artigo aborda o documentário interativo produzido na Ibero-América a partir de um estudo exploratório-descritivo nos últimos cinco anos. A interatividade vai para além de uma contribuição seletiva e participativa em direção a um impacto de transformação social num determinado contexto. A proximidade, como foco das histórias no documentário interativo ibero-americano, leva a experiências e iniciativas que convidam a mudanças e ações, com suporte num forte caráter social.

Palavras-chave: documentário interativo; Ibero-América; proximidade; transformação.

Resumen: Este artículo aborda el documental interactivo producido en Iberoamérica a partir de un estudio exploratorio-descriptivo en los últimos cinco años. La interactividad va más allá de una contribución selectiva y participativa, hacia el impacto y la transformación social en un entorno. La proximidad, como foco de las historias en el documental interactivo iberoamericano, conduce a experiencias e iniciativas que invitan al cambio y la acción, basadas en un fuerte carácter social.

Palabras clave: documental interactivo; Iberoamérica; proximidad; transformación.

Abstract: This article deals with the interactive documentary produced in Ibero-America from an exploratory-descriptive study in the last five years. Interactivity goes beyond a selective and participatory contribution towards the impact and social transformation in an environment. Proximity, as a focus of the stories in the Ibero-American interactive documentary, leads to experiences and initiatives that invite change and action, based on a strong social character.

Keywords: interactive documentary; Ibero-America; proximity; transformation.

Résumé : Cet article traite du documentaire interactif produit en Amérique Latine à partir d'une étude exploratoire-descriptive des cinq dernières années. L'interactivité va au-delà d'une contribution sélective et participative à l'impact et à la transformation sociale dans un environnement. La proximité, en tant que point focal des histoires du documentaire interactif ibéro-américain, conduit à des expériences et des initiatives qui invitent au changement et à l'action, basées sur un fort caractère social.

Mots-clés : documentaire interactif ; Ibéro-Amérique ; proximité ; transformation.

* Jorge Vázquez-Herrero: Universidade de Santiago de Compostela, Facultad de Ciencias de la Comunicación, Grupo de Investigación Novos Medios. 15782 Santiago de Compostela, España. E-mail: jorge.vazquez@usc.es

Gisela Moreno: Universidad Nacional de Rosario, Facultad de Ciencias Políticas y Relaciones Internacionales, Escuela de Comunicación Social. 1065, S2000CGK Rosario, Santa Fe, Argentina. E-mail: giselamgm@gmail.com

Doc On-line, n. especial, dezembro de 2017, www.doc.ubi.pt, pp. 109-130. 


\section{Introducción}

El documental interactivo, que ha evolucionado en las últimas décadas sin llegar a alcanzar una producción masificada a nivel global, toma un camino de crecimiento en las realidades cercanas con necesidad de ser representadas. Gifreu-Castells (2017) destaca la producción en Latinoamérica, donde varios factores entran en juego: los documentales son realizados a menudo desde un punto de vista emocional, hay una lengua común, potencial para interconectar proyectos locales y oportunidades en las representaciones históricas. En este artículo se aborda la producción iberoamericana de documental interactivo entre 2013 y 2017. Una mirada sobre el impacto social directo y potencial de los proyectos seleccionados permite concluir con el establecimiento del formato como un elemento integrador y de visibilización.

\section{Marco teórico}

En el escenario actual de la comunicación, la interactividad y la participación son conceptos comunes en un debate alimentado por el desarrollo de los medios digitales. La convergencia ha supuesto transformaciones constantes en diversos campos como la aplicación de la tecnología o el papel de las audiencias. La interactividad se ha convertido en la característica más significativa del nuevo panorama, potenciando así las posibilidades de determinados productos.

En el caso del documental, mantiene su carácter audiovisual pero incorpora nuevos lenguajes y formatos en el medio interactivo. Además de representar la realidad, emplea diferentes modalidades para navegar e interactuar (Gifreu, 2013), abandonando la autoría exclusiva del documentalista y cediendo control al usuario. Se establece una nueva relación entre el autor, el texto y el usuario, hacia una autoría compartida (Nash, 2012; Favero, 2013). El cambio supone a su vez un rol más activo del público (Aston y Gaudenzi, 2012), lo que favorece la experiencia individual, protagonista o personalizada.

El desarrollo del documental interactivo, muy ligado a la Web, ha provocado que se le identifique también como webdoc o documental web. Sin embargo, existen mútiples denominaciones con leves diferencias, como el idoc o el documental transmedia. En los últimos años, también ha avanzado la exploración y caracterización del género con importantes contribuciones de investigadores de todo el mundo (Miles, 2014; Nash, Hight y Summerhayes, 2014; Gantier y Labour, 2015; Aston, Gaudenzi y Rose, 2017), lo que evidencia su actualidad e interés. 
En sus más de treinta años de evolución (Gifreu, 2013), ha tomado la forma de una obra de no ficción que representa un fragmento de la realidad a través de un medio interactivo, siendo esta una definición abierta que permite abarcar su complejidad y diversidad. Gaudenzi (2013) apuntó hacia un documental interactivo más evolucionado, el living documentary, que se va transformando con el paso de los usuarios, una idea disruptiva que refleja su potencial. Por otra parte, Nash (2014) se refiere a dos tipos de participación: voz como autor y voz como participación social, siendo la primera la que se relaciona con tomar parte en la realización y la segunda la que considera la relación con otros usuarios - una participación a través de la obra-. Otros autores, como Eva Domínguez (2013), señalan que se trata de una forma del periodismo inmersivo: "el grado de implicación del usuario aumenta por la toma de decisiones, la ejecución de un rol y la posibilidad de actuar en el escenario" (Vázquez-Herrero et al., 2017).

Tras una primera definición del documental interactivo a día de hoy, nos centramos en el valor de la interactividad y la participación en este contexto. La cultura participativa fue definida por Jenkins (2006) como aquella en la que "los fans y otros consumidores son invitados a participar activamente en la creación y circulación de nuevo contenido", mientras que la interactividad se basa en la interacción usuario-contenido y usuario-usuario (Carpentier, 2015). Por tanto, la participación integra diferentes tipos de interacción, favoreciendo un nivel más alto de compromiso cívico y social (Cortés-Selva y Pérez-Escolar, 2016).

Previamente, se ha estudiado el impacto del documental interactivo en áreas como la participación política ciudadana, donde Cortés-Selva y PérezEscolar (2016) destacan la capacidad de transformar al ciudadano pasivo en actor político. El activismo transmedia como vía de participación social es abordado por Ortuño y Villaplana, quienes identifican distintas estrategias en el documental interactivo como "formas de acción que entretejen procesos participativos físicos, mentales o sociales" (2017).

Dicha participación, con sus diferentes mecanismos, se vuelve más significativa cuando el usuario alcanza la figura de co-creador, por ejemplo, al generar contenido para la propia obra o tomar parte en la expansión del relato. De esta forma, la co-creación en el documental interactivo es considerada "una ruta para establecer diálogos que pueden proveer recursos significativos en los procesos de cambio en marcha" (Rose, 2017).

A partir del estudio de caso de 0 responsables (Barret Films, 2013), Calatayud (2015) señala la necesidad de ampliar los estudios sobre el webdoc desde una perspectiva social más allá de la plataforma virtual, siendo esta una 
de las premisas fundamentales para considerar en este estudio la estrategia transmedia. Sobre este aspecto, aplicado al documental, han trabajado diversos autores. Entre ellos, Renó (2013) concuerda con Calatayud al indicar que "el documental transmedia no exige una circulación exclusivamente por ambientes digitales, pues se trata de un lenguaje, una manera de contar una historia". Por otra parte, el usuario se vuelve central. Las audiencias activas articulan las diferentes plataformas, fluyen entre ellas, les aportan valor, generan nuevos espacios y actúan en lo virtual y lo real:

El transmedia storytelling necesita que los actores participantes estén dispuestos a interactuar y formar parte de la trama. Que tengan un pleno compromiso con la narración: ser 'ciudadanos' que habiten y recorran las historias que no sólo se desarrollan en escenarios virtuales sino en el territorio real. (Irigaray, 2015a).

En cuanto a la producción, el desarrollo de formatos de no ficción interactiva, y concretamente el documental interactivo transmedia, están vinculados a la experimentación y la innovación, disociado de un modelo de negocio rentable inmediato e impulsado por otros objetivos. Esto provoca que la creación prolifere fuera del sistema de medios tradicionales, como así señala Fernando Irigaray, experto en la dirección de proyectos transmedia desde la Universidad Nacional de Rosario:

Producir transmedia desde la periferia significa correrse de la centralidad no sólo de la gran financiación de productos culturales industriales, es adentrarse en temas complejos que se alejan de la estigmatización de los medios tradicionales, entender a la ciudad como contenedora de múltiples historias que componen universos narrativos, que atraviesan diversos productos en diferentes pantallas, medios y acciones territoriales, traccionando audiencias y usuarios entre sí. (Irigaray, 2015b).

\section{Metodología}

Los vínculos existentes entre los países iberoamericanos, y su diversidad cultural, justifican el interés de las investigaciones que plantean una aproximación conjunta o un estudio comparativo. Además de las cuestiones históricas, las lenguas compartidas - española y portuguesa - suponen una importante ventana para la difusión del contenido producido dentro de la región.

El documental interactivo y transmedia, como forma de expresión de la no ficción interactiva, todavía se considera un objeto de estudio novedoso debido a la reducida literatura específica en el campo. Por esta razón, un enfoque ubicado sobre la producción de documental interactivo y transmedia en Iberoamérica y centrada en los espacios alternativos, sugiere la necesidad de una exploración. 
El estudio exploratorio-descriptivo es la técnica empleada para identificar las principales características de los productos bajo la denominación de documental interactivo y transmedia, conforme a los antecedentes revisados, en el periodo desde 2013 a 2017 en los países iberoamericanos. Con el universo de la producción de este formato como referencia, el seguimiento de la actualidad y el rastreo de bases de datos, eventos, premios y convocatorias, da lugar a una muestra de 46 productos. Posteriormente, se seleccionan 12 producciones para analizar la construcción del documental interactivo y transmedia, así como su contribución a la transformación social en la proximidad. Se trata de una muestra de documentales interactivos producidos fuera del sistema de medios y de las grandes productoras internacionales, poniendo así en valor otros espacios alternativos de creación y difusión.

En primer lugar, se describe en cada documental la temática y su ámbito geográfico, en cuanto a la dimensión que abarca la realidad representada, situándola en el ámbito hiperlocal, local, regional, nacional, continental o global. Por otra parte, se analizan la propuesta interactiva y los mecanismos de participación. En una segunda fase del estudio, se realiza una valoración cualitativa del documental como elemento activo frente a un entorno social: un motor de identidad, cambio y movilización desde lo pequeño y cotidiano. Se identifican las llamadas a la participación del usuario, las actuaciones de la propia producción sobre el terreno y el impacto potencial del proyecto sobre el entorno.

Tabla 1. Selección de documentales interactivos 2013-2017

\begin{tabular}{lll} 
Título & Año de producción & País de origen \\
\hline O responsables & 2013 & España \\
El charco azul & 2013 & Colombia \\
Calles perdidas & 2013 & Argentina \\
Chaco-PY & 2014 & Paraguay \\
Illados & 2014 & España \\
4 ríos & 2014 & Colombia \\
Mujeres en venta & 2015 & Argentina \\
Proyecto Quipu & 2015 & Chile, Perú, Reino Unido \\
Pregoneros de Medellín & 2015 & Colombia \\
Canción de la Ciudad & 2015 & Argentina \\
Desiguales & 2016 & Argentina \\
El feriante & 2017 & Argentina \\
\hline
\end{tabular}

Elaboración propia 


\section{Contexto iberoamericano de producción del documental interactivo}

El escenario actual en el que se desarrolla el documental interactivo en Iberoamérica está asentado sobre algunos antecedentes clave. Como suele suceder, la iniciativa de los pioneros y de los primeros seguidores dan pie a la consolidación, en este caso, de una nueva forma de retratar la realidad. En la década del 2000, importantes medios de la región como El País Cali (Colombia) y Clarín (Argentina) experimentaban con el formato de especial: Cali, la ciudad que no duerme (El País Cali, 2009), Cayucos (Clarín, 2009), La hoja sagrada (El País Cali, 2010), Ay México lindo: la narcoguerra (Clarín, 2010). Por otra parte, en el ámbito universitario, el proyecto DocuMedia de la Universidad Nacional de Rosario (Argentina) producía Vibrato (2008), Obras en construcción (2009) y Migraciones (2011) y el Instituto Tecnológico de Monterrey (México) en colaboración con la Universidad de Carolina del Norte en Chapel Hill (Estados Unidos) presentaba Repensando México (2011).

Los proyectos nacidos a finales de la pasada década demostraban el potencial multimedia del medio digital y, en cierta medida, una interactividad que permitía planteamientos alternativos de la historia, pasando por la no linealidad que facilitaba un visionado más personal en cuanto a ritmo, orden y profundidad. La principal tecnología empleada para el desarrollo de estos formatos era Flash, que se vio superado por la transición al HTML5. El avance en el estándar del lenguaje de marcado ha permitido que los contenidos sean más accesibles y adaptables a diferentes dispositivos, condición fundamental para su distribución en el contexto multipantalla actual.

Los países latinoamericanos no solo han estado presentes como productores en los primeros años de desarrollo del documental interactivo, o la forma híbrida próxima al especial multimedia que se identifica en la década anterior, sino que han sido protagonistas de historias producidas desde otros países. Uno de los casos más significativos es el de Argentina, pues ahí han puesto su mirada grandes productores como el canal franco-alemán Arte - Argentina (2010) - y National Film Board of Canada - Primal (2014) -. Radiotelevisión Española produjo En el reino del plomo (2013) en Honduras; Arte, Upian y Vu crearon Alma, hija de la violencia (2012) enmarcado en Guatemala; y The Martin Agency junto a la Biblioteca Presidencial John Fitzgerald Kennedy, Clouds Over Cuba (2012).

Otro de los factores que influyen en el desarrollo del documental interactivo en Iberoamérica es la organización de eventos, foros y congresos relacionados con la no ficción interactiva, específicamente o adaptando sus líneas de interés. A partir del Festival DocsBarcelona, se celebran DocsBarcelona Medellín en Colombia desde 2013 y Docs Barcelona Valparaíso en Chile desde 
2016. InterDocsBarcelona, las actividades interactivas y transmedia del festival DocsBarcelona, han generado en Colombia y Chile desde 2014 una gran variedad de eventos interactivos tales como conferencias, pitch interactivos, hackatones, talleres de desarrollo interactivos y presentación de estudios de caso. En Argentina se ha celebrado en 2016 el I Encuentro de Narrativas Documentales Transmedia, a cargo de Laboratorio 22, y el congreso Visible Evidence XXIV en la ciudad de Buenos Aires en 2017. En España se celebran, además del mencionado festival de documental en Barcelona, el Encuentro de creadores y profesionales de la narrativa digital y el documental interactivo (\#hacerwebdoc) como parte del festival DocumentaMadrid - tercera edición en 2017 -, así como diferentes congresos académicos que integran la no ficción interactiva, como el Congreso Internacional de Nuevas Narrativas, organizado por la Universitat Autònoma de Barcelona, en su primera edición de 2017. A las diferentes actividades que se desarrollan hay que añadir el Laboratorio Audiovisual Interactivo en Cali (Colombia) - tercera edición en 2017 - o el Concurso de documental interactivo organizado por la Pontificia Universidad Javeriana (Colombia) junto a Distrito Cinema, con dotación económica para formación.

Por último, un tercer punto fundamental es el apoyo institucional y la financiación disponible para el desarrollo de proyectos. En España, Radiotelevisión Española lanzó en 2016 una convocatoria de coproducciones denominada Factoría de webdocs, que dio lugar a seis nuevos documentales interactivos, entre ellos: Párkinson, que tiemble el camino (2016), Memoria de futuro (2017) y Guerra a la mentira (2017). Sin embargo, la televisión pública catalana ya había experimentado con este modelo con anterioridad, dando lugar a obras como Amb títol (2016) o Orgull de Baix (2016). Desde el gobierno español, se promueven las Ayudas para la modernización e innovación de las industrias culturales y creativas - sector profesional - y las Ayudas de acción y promoción cultural - personas jurídicas sin ánimo de lucro -, dentro del Plan de fomento de las industrias culturales y creativas 2016. En ambos casos se sugiere la creación de contenidos digitales, junto a otros objetivos estructurales. Colombia cuenta con diferentes convocatorias, apostando por la innovación en los medios, como Crea Digital ${ }^{1}$ o la Convocatoria de estímulos del Ministerio de Cultura con partidas específicas para las narrativas transmedia y el documental expandido. En Argentina, el Instituto Nacional de Cine y Artes Audiovisuales (INCAA) incorpora en los concursos nacionales del Plan de fo-

1. En 2017, el programa Crea Digital, del Ministerio de Tecnologías de la Información y las Comunicaciones y el Ministerio de Cultura de Colombia, abrieron una convocatoria para proyectos de contenidos digitales con un presupuesto aproximado de 565.000 euros. Desde 2012, han financiado 16 proyectos transmedia. 
mento para la producción de contenidos la dimensión transmedia desde 2016. Asimismo, se están desarrollando proyectos de documental transmedia en este país a través de los fondos de la Red Nacional Audiovisual Universitaria (RENAU) del Consejo Interuniversitario Nacional (CIN).

\section{Casos de estudio}

0 responsables es un documental web seriado que recupera la investigación sobre el accidente de metro el 3 de julio de 2006 en Valencia. A través de seis capítulos, revisa las diferentes perspectivas del fatal suceso. Fue producido en 2013 por la cooperativa Barret Films junto a la Asociación de víctimas del metro del 3 de julio, con el apoyo de la Fundació Valencianista i Demòcrata Josep Lluis Blasco. El principal aporte interactivo se identifica en una plaza virtual donde los usuarios pueden asistir a la concentración, simulando la que se realiza mensualmente cada día 3 en la Plaza de la Virgen de Valencia, a través del login con Twitter o Facebook. Asimismo, pueden participar en la investigación incompleta con documentos, comentarios o archivos audiovisuales y en la recogida de firmas por una investigación veraz en Change.org. En su estrategia transmedia contempla el documental audiovisual lineal, La estrategia del silencio (2017), dirigido por Vicent Peris.

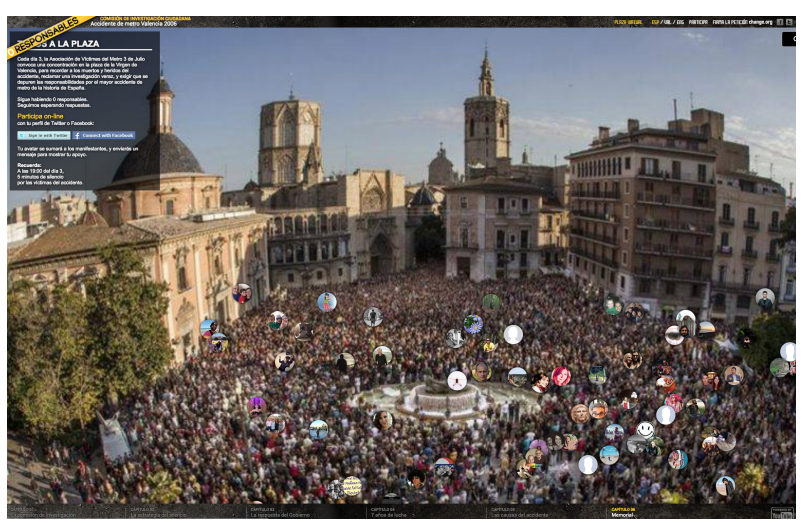

Plaza virtual en 0 responsables.

Fuente: Oresponsables.com

En El charco azul, una comunidad local en Buenaventura (Colombia) muestra su visión de la realidad y revaloriza la cultura oral afro. Cuenta con seis partes conectadas por una ruta ferroviaria abandonada, a través de la que el usuario puede conocer diferentes historias, personajes, galerías fotográficas y playlists. La producción corrió a cargo de Ojo de Pez y Señal Colombia en 
2013. La interactividad es principalmente selectiva al permitir un acceso no secuencial a los contenidos; la participación se limita a las redes sociales.

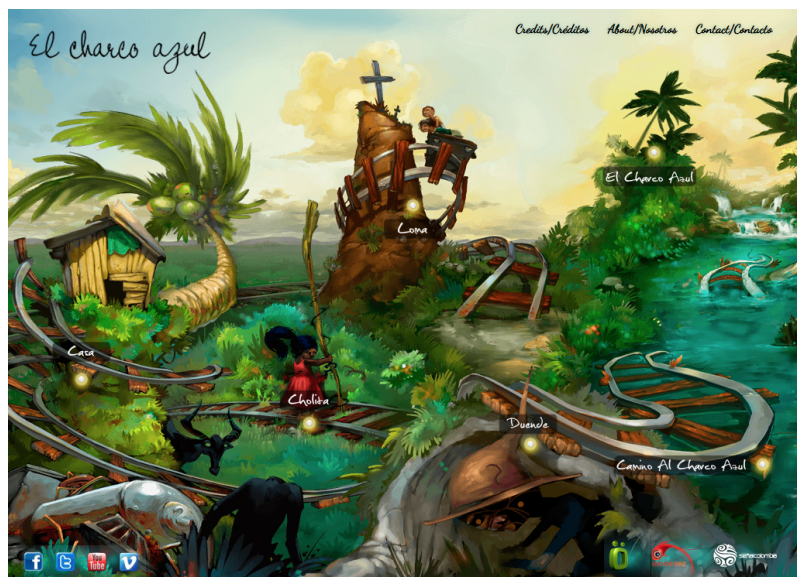

Menú de navegación en El charco azul.

Fuente: elcharcoazul.co

Calles perdidas aborda el narcotráfico en la ciudad de Rosario (Argentina) desde diferentes perspectivas como la política, la circulación de la droga y la economía. En seis apartados temáticos se estructura este relato documental centrado en la localidad argentina. Producido en 2013 por la Dirección de Comunicación Multimedial de la Universidad Nacional de Rosario y declarado de interés municipal. El usuario puede elegir el contenido específico que quiere visualizar o participar con comentarios a través de Facebook. 


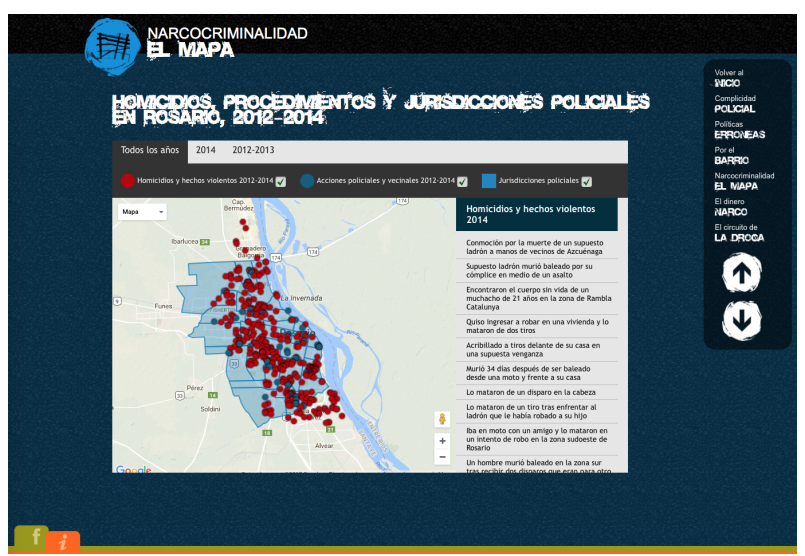

Mapa del narcocrimen en Calles perdidas.

Fuente: documedia.com.ar/callesperdidas

El documental Chaco-PY trata sobre el presente y el futuro de la región del Chaco paraguayo, una zona de alta deforestación. Se estructura en cinco bloques interconectados con contenidos más específicos alrededor de cada concepto. Fue creado por Andrea Ruffini en 2014 en Paraguay. La interactividad selectiva es protagonista por la propuesta de estructura reticular del proyecto, sin embargo, la participación queda relegada a la página en Facebook.

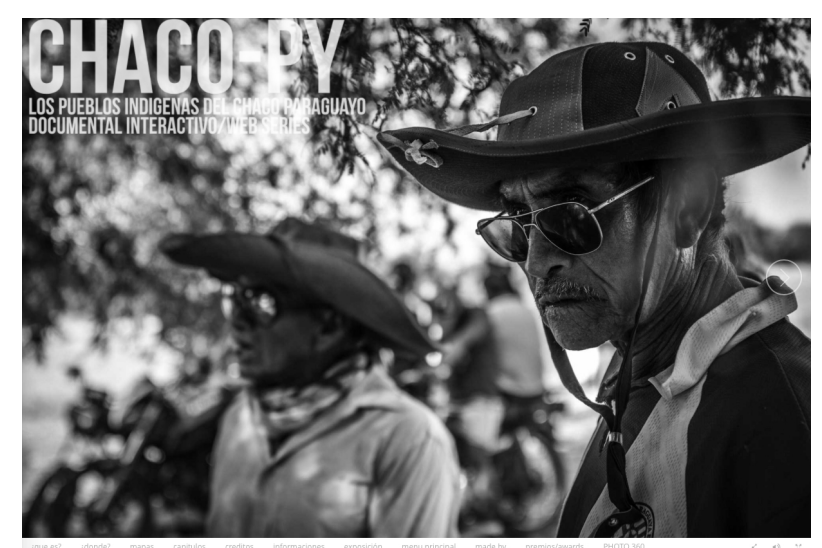

Portada de Chaco-PY.

Fuente: chaco-py.com

Illados contribuye a la memoria colectiva con cinco historias de estilos de vida en extinción, situándose en Galicia (España). Producido en 2014 por 
Laura Santos, Sibila Souto y Jorge Vázquez, este documental trata de poner en valor las historias adaptándolas a las nuevas pantallas. Los usuarios pueden participar a través de redes sociales y del formulario final en el que pueden enviar su propio relato o contribución.

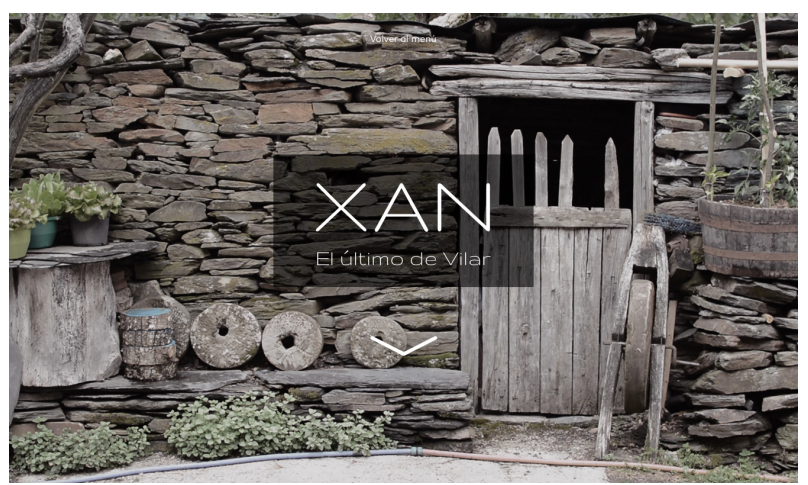

Xan en Illados.

Fuente: illados.com

El proyecto transmedia 4 ríos incluye, además del webdoc, un cómic, una maqueta de realidad aumentada y un cortometraje para abordar las consecuencias del conflicto armado colombiano durante los últimos 40 años. Fue producido en 2014 por Orgánica Digital en Colombia. Está estructurado en base a una línea principal con contenidos extra interrelacionados, además de un espacio para la participación a través de texto, audio o dibujo en el "flujo de memoria", o bien, en las redes sociales.

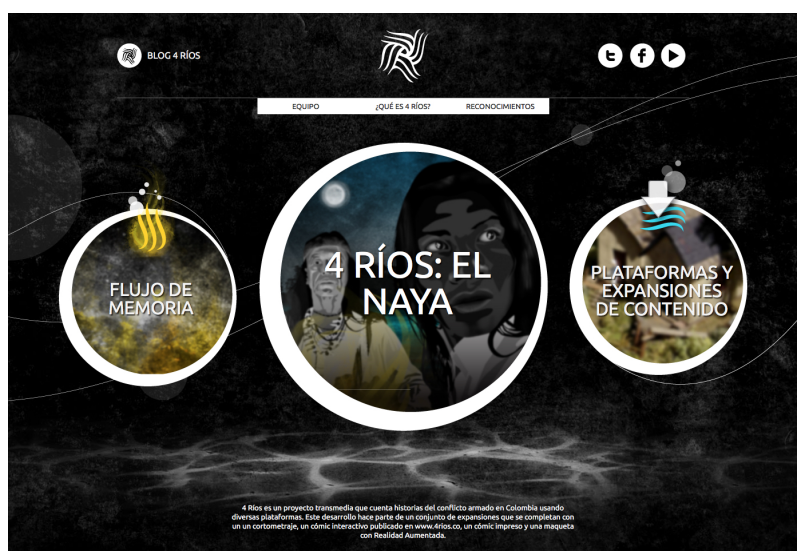

Portada de 4 ríos. Fuente: 4rios.co 
Mujeres en venta se presenta como documental transmedia sobre la trata de personas con fines de explotación sexual en Argentina, a través de un recorrido de cinco capítulos desde la captación, las rutas y el rescate de las víctimas. Este proyecto fue producido en 2015 por la Dirección de Comunicación Multimedial de la Universidad Nacional de Rosario. Los usuarios participan en las redes sociales y a través de un mapa interactivo en el que incorporar hechos relacionados con la temática del webdoc, tomando así parte en el relato.

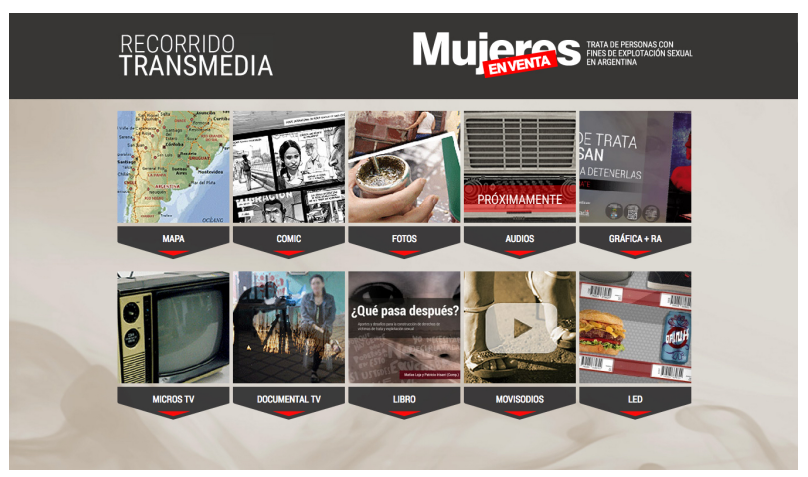

Universo transmedia en Mujeres en venta. Fuente: documedia.com.ar/mujeres

Proyecto Quipu aborda las esterilizaciones forzadas a cargo del gobierno peruano en los años noventa, dando voz a las víctimas y reclamando justicia ante los sucesos no reconocidos oficialmente. El documental fue publicado en 2015 y producido por Chaka Studio, involucrando tres países: Perú, Chile y Reino Unido. Estructurado como un quipu, donde los incas registraban y contabilizaban, presenta los mensajes de las personas afectadas y de los usuarios que pueden dejar su respuesta en el webdoc, aportar una donación, contribuir como voluntarios en el proyecto o firmar una petición dirigida al presidente del país. 


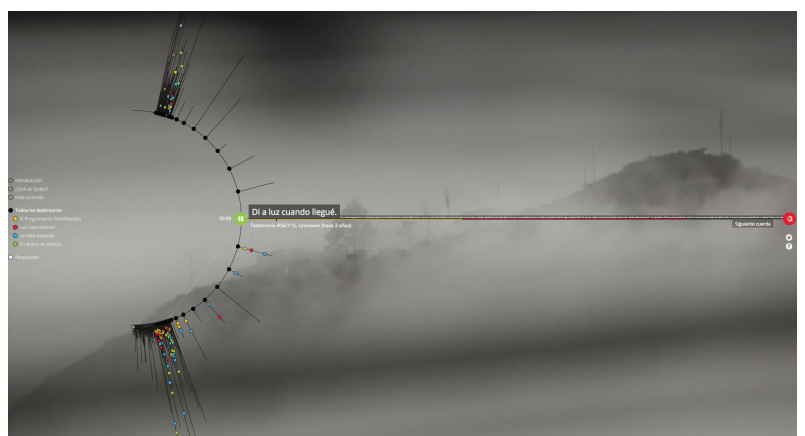

Navegación inspirada en un quipu, en Proyecto Quipu.

Fuente: quipu-project.com

En Pregoneros de Medellín, el usuario recorre las calles de la ciudad colombiana conociendo a los vendedores ambulantes que llaman la atención de los viandantes con sus canciones. Producido en 2015 por el grupo creativo Carabalí junto a Thibault Durand, presenta cinco personajes a través de un recorrido virtual por Medellín, donde el usuario debe ir desbloqueando contenidos asociados con un planteamiento ludificado. No hay opciones de interactividad participativa en el propio documental.

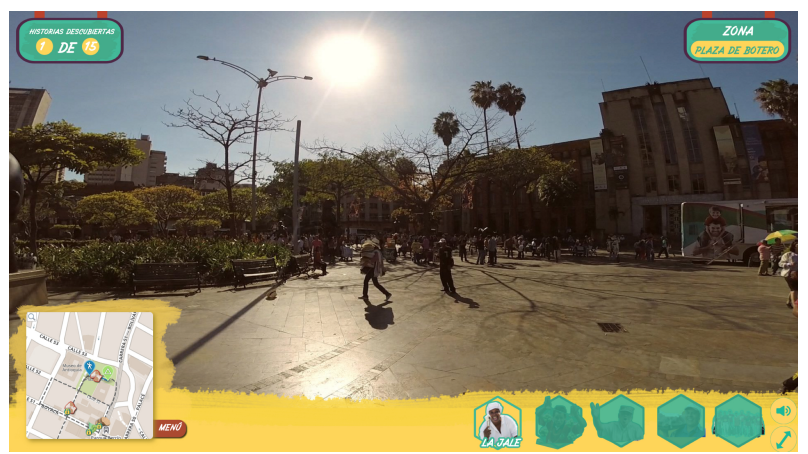

Recorriendo el centro urbano de Medellín, en Pregoneros de Medellín.

Fuente: pregonerosdemedellin.com

Canción de la Ciudad es un webdoc sobre los músicos callejeros de la ciudad de Rosario, en Argentina. Producido en 2015 por la Facultad Libre, con sede en dicha ciudad, ofrece ocho capítulos con las historias de los artistas, además de contenidos para otros soportes narrativos. El papel del usuario se centra en la navegación del webdoc y la contribución con vídeos generados por el público en la sección "prosumidores". 


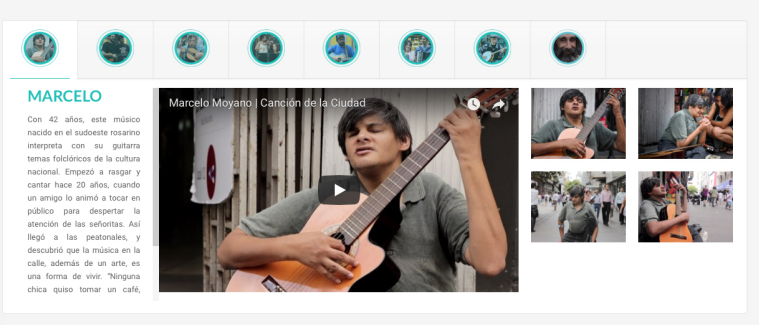

Menú de historias en Canción de la Ciudad. Fuente: cancionciudad.com

Desiguales se centra en las desigualdades y diferencias entre los países latinoamericanos, desde múltiples puntos de vista. Nace en 2015, de la mano de la Cátedra Latinoamericana de Narrativas Transmedia, como un proyecto colaborativo. El usuario puede participar elaborando su propia mirada en las diferentes categorías que consideran: webisodios, proyecciones callejeras, realidad aumentada o presentaciones; y geolocalizarla sobre el mapa que da acceso al contenido.

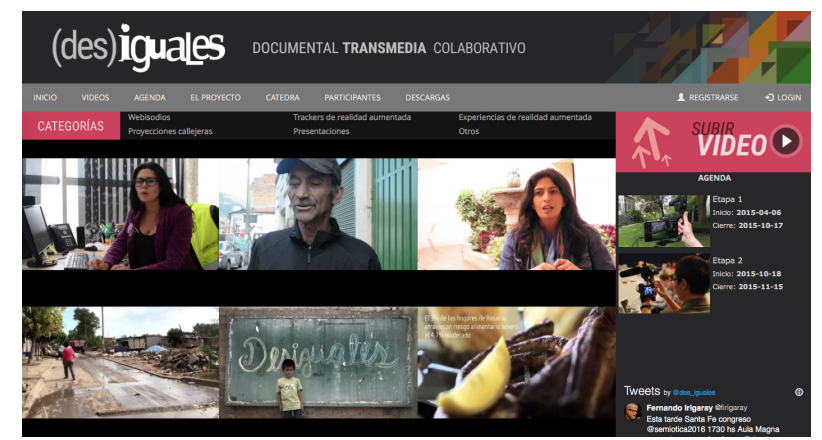

Vídeos del documental transmedia colaborativo Desiguales. Fuente: des-iguales.com

Por último, El feriante aporta una visión de la situación de encierro y la vida posterior a la prisión en Argentina, haciendo visible el papel de los talleres de formación y producción cultural en las cárceles de Rosario. Fue producido en 2017 por la Facultad Libre y el colectivo La Bemba del Sur. Desde el punto de vista del usuario, la interactividad es selectiva, permitiendo un consumo individualizado de los cinco capítulos del webdoc y el conjunto de soportes alternativos. La participación se canaliza en las redes sociales. 


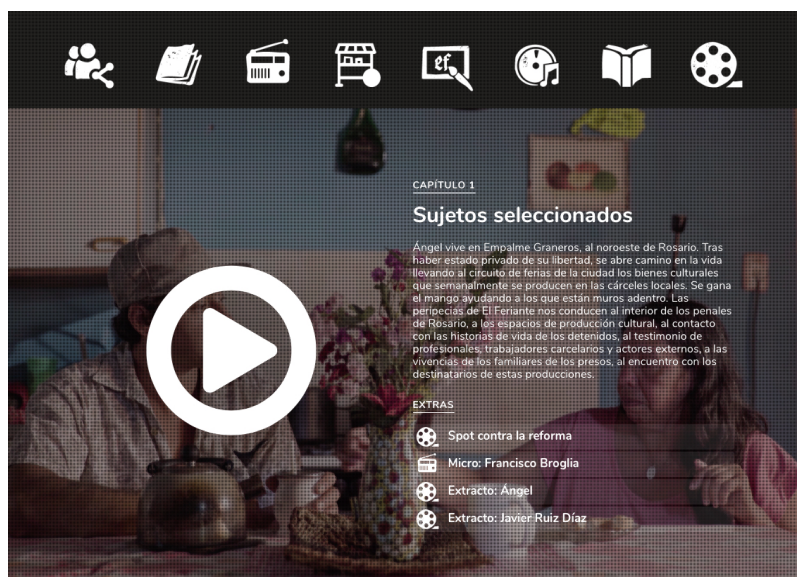

Menú de plataformas y capítulo 1 de El feriante.

Fuente: elferiante.com.ar

\section{Análisis temático y de ámbito geográfico}

Tras esta descripción de los proyectos y de su propuesta de interactividad, se considera relevante atender a la temática y al ámbito geográfico de cada uno de los casos. Los documentales interactivos seleccionados tratan, por este orden: la denuncia, los estilos de vida y la cultura. Dentro de los enfoques de denuncia social se incluyen razones de memoria, de conflicto o violencia y de integración. En general, el protagonismo de los relatos se concentra en áreas sociales e historias personales.

Respecto al ámbito geográfico en el que se desarrolla el documental, se identifica un predominio de las cuestiones locales - en la mitad de los casos seleccionados -, seguido del ámbito nacional, regional y continental. Este dato refleja el interés por los espacios más próximos y específicos, como la ciudad o el barrio. 
Tabla 2. Clasificación temática y de ámbito geográfico en la muestra de

\begin{tabular}{|c|c|c|c|}
\hline Título & Tema específico & Temática general & Ámbito geográfico \\
\hline 0 responsables & $\begin{array}{l}\text { Responsabilidad } \\
\text { política }\end{array}$ & $\begin{array}{l}\text { Denuncia y } \\
\text { memoria }\end{array}$ & $\begin{array}{l}\text { Local (Valencia, } \\
\text { España) }\end{array}$ \\
\hline El charco azul & Cultura afro & Estilos de vida & $\begin{array}{l}\text { Local (Buenaventu- } \\
\text { ra, Colombia) }\end{array}$ \\
\hline Calles perdidas & Narcotráfico & $\begin{array}{l}\text { Denuncia y } \\
\text { conflicto }\end{array}$ & $\begin{array}{l}\text { Local (Rosario, } \\
\text { Argentina) }\end{array}$ \\
\hline Chaco-PY & Pueblos indígenas & Estilos de vida & $\begin{array}{l}\text { Regional (Chaco, } \\
\text { Paraguay) }\end{array}$ \\
\hline Illados & Estilos de vida & Estilos de vida & $\begin{array}{l}\text { Regional (Galicia, } \\
\text { España) }\end{array}$ \\
\hline 4 ríos & Conflicto armado & $\begin{array}{l}\text { Denuncia y } \\
\text { memoria }\end{array}$ & $\begin{array}{l}\text { Nacional } \\
\text { (Colombia) }\end{array}$ \\
\hline Mujeres en venta & $\begin{array}{l}\text { Explotación } \\
\text { sexual }\end{array}$ & $\begin{array}{l}\text { Denuncia y } \\
\text { conflicto }\end{array}$ & $\begin{array}{l}\text { Nacional } \\
\text { (Argentina) }\end{array}$ \\
\hline Proyecto Quipu & $\begin{array}{l}\text { Esterilizaciones } \\
\text { forzadas }\end{array}$ & $\begin{array}{l}\text { Denuncia y } \\
\text { memoria }\end{array}$ & Nacional (Perú) \\
\hline Pregoneros de Medellín & Venta ambulante & Estilos de vida & $\begin{array}{l}\text { Local (Medellín, } \\
\text { Colombia) }\end{array}$ \\
\hline Canción de la Ciudad & Música callejera & Cultura & $\begin{array}{l}\text { Local (Rosario, } \\
\text { Argentina) }\end{array}$ \\
\hline Desiguales & Desigualdades & $\begin{array}{l}\text { Denuncia y } \\
\text { conflicto }\end{array}$ & $\begin{array}{l}\text { Continental } \\
\text { (Sudamérica) }\end{array}$ \\
\hline El feriante & Integración & $\begin{array}{l}\text { Denuncia e } \\
\text { integración }\end{array}$ & $\begin{array}{l}\text { Local (Rosario, } \\
\text { Argentina) }\end{array}$ \\
\hline
\end{tabular}

Elaboración propia

\section{Análisis del impacto social}

En el análisis introductorio se identificaron los principales mecanismos interactivos, sin embargo, estos documentales presentan objetivos enfocados a la transformación social. Por una parte, hay una serie de acciones que buscan un impacto social directo - llamadas a la acción, iniciativas y actuaciones sobre el terreno, que son llevadas a cabo por el propio equipo de producción- y otras de tipo indirecto -impacto potencial en el entorno como resultado de la difusión de la obra -.

Entre las diferentes interpelaciones que se lanzan al público, destacan dos casos especialmente significativos. Proyecto Quipu establece una comunica- 
ción bidireccional entre las víctimas y los usuarios, una conversación que trasladan al mundo real y representan a través del webdoc. Se convierte así en una potente herramienta tanto para las comunidades de afectados como para quien establece un contacto con las esterilizaciones forzadas en la década de los noventa en Perú. Otro ejemplo que conecta la realidad física con la representación virtual es la llamada a concentrarse para demandar justicia de 0 responsables ${ }^{2}$. Los usuarios son invitados a participar del encuentro que se produce mensualmente en Valencia o a unirse en la concentración virtual, donde aparecen representados a través de su perfil de Twitter o Facebook dentro del webdoc.

En la muestra de documentales interactivos analizados, el público puede participar en proyectos colaborativos como Desiguales o Canción de la Ciu$d a d$, con su propio contenido audiovisual, o aportando información para la investigación en 0 responsables. Hay mensajes dirigidos a que tomen acción denunciando y sumándose a un cambio en la sociedad - Mujeres en venta -, a contribuir en la memoria y en el debate -4 ríos, Illados -. La obra documental no solo representa una realidad sino que trata de establecer vínculos con ella e invita a tomar parte. Asimismo, se localizan diferentes iniciativas asociadas a estos proyectos: presentaciones públicas, exhibiciones, talleres, exposiciones, festivales, espacios de información y recogidas de firmas. Sobre este último punto, 0 responsables consiguió 128.642 firmas en su petición dirigida al Presidente de la Generalitat Valenciana y Proyecto Quipu empleó la plataforma del diario La República, aunque no constan resultados, para hacerle llegar al Presidente de Perú su demanda contra el crimen de lesa humanidad que denuncian.

Para la realización de algunos de estos documentales, fue necesaria una intervención sobre el terreno y el desarrollo de actividades más allá de la producción en sí misma. Por ejemplo, detrás de Proyecto Quipu hay un trabajo de campo inicial para crear confianza con la tecnología que se implementaba. Se trataba de introducir dispositivos de telefonía VOIP en áreas de muy baja penetración de Internet, planteando así un reto de inclusión más allá del objetivo principal del documental, para lo que contaron también con la colaboración de radios locales que difundieron el proyecto que se estaba llevando a cabo. Las relaciones con otras instituciones pueden ser estratégicas para la difusión del documental, pero también para ser representadas y visibilizadas, como ocurre

2. Ortuño y Villaplana (2017) destacan la contribución de las diferentes acciones que se sucedieron y que encaminaron las reacciones, además del webdoc: "Como hemos visto, la denuncia televisiva en un programa de máxima audiencia, la aparición de noticias sobre el caso en la prensa tradicional, y sobre todo la infatigable lucha en los tribunales de la asociación de familiares de las víctimas han sido, junto con el webdoc, partes necesarias para la reapertura del caso y la involucración en este de buena parte de la sociedad, principalmente de la valenciana". 
con la Asociación de víctimas del metro del 3 de julio en 0 responsables o La Bemba del Sur en El feriante. La actuación sobre el terreno supone también participar en actividades públicas e incluso emplear el espacio urbano como un lugar de exhibición. De esta forma, Mujeres en venta empleó el territorio para expandir sus soportes narrativos, a través de gráficas con realidad aumentada en las calles de Rosario.

Finalmente, el documental interactivo está impactando en el entorno de forma directa y con un carácter potencial que, a medio y largo plazo, puede suponer las transformaciones demandadas o al menos un paso hacia adelante en el debate público, la visibilidad de las temáticas, la denuncia, la concienciación, la integración en sociedad de colectivos marginados y de minorías o la dignificación de oficios. En algunos de los casos analizados existen evidencias o resultados específicos, como las peticiones masivas ${ }^{3}$ o la repercusión en medios de comunicación ${ }^{4}$.

\section{Cooperación y expansión transmedia: el caso DocuMedia}

Cuando en 2008 desde el equipo de la Dirección de Comunicación Multimedial de la Universidad Nacional de Rosario se comenzó a experimentar con el formato de especial multimedia interactivo, al que se denominó DocuMedia, se decidió desde el inicio enfocar los desarrollos en problemáticas próximas, de alcance local, en las cuales una institución pública como la universitaria pudiera aportar su mirada pero también pudiera funcionar como articulador de grupos, organizaciones y equipos que se encontraran trabajando en pos de generar cambios sociales en estos contextos. Se trabajó con diversas ONGs, asociaciones civiles, organizaciones políticas y sociales, así como equipos de trabajo de distintos estamentos del estado (municipal, provincial y nacional) tanto en el caso de Calles Perdidas como en Mujeres en Venta.

Esto implicó un impacto mayor respecto al que el equipo de producción podría haber provocado en materia de divulgación, concienciación, denuncia o acciones llevadas a cabo sobre el territorio. Las organizaciones, que ya se encontraban realizando trabajo barrial militante en torno a las problemáticas

3. 0 responsables. La Asociación de víctimas del metro del 3 de julio lanzó una campaña en change.org que terminó con éxito. A día de hoy todavía actualizan la información en ese espacio, donde 128.642 personas firmaron. (https://www.change.org/p/pide-al-president-fabrauna-investigación-veraz-sobre-el-accidente-de-metro-de-2006)

4. Diversos medios se hicieron eco del documental Proyecto Quipu; entre ellos, El País (https://elpais.com/elpais/2016/04/28/planeta_futuro/1461846356_024411.html) y la BBC (www.bbc.com/mundo/noticias-america-latina-39413935). El debate público de estos hechos ha llevado a la movilización y la denuncia, como apuntan también diferentes medios, entre ellos Clarín (www.clarin.com/mundo/denuncian-alberto-fujimori-esterilizacion-forzosa236-mil-mujeres_0_rJXFauz2W.html). 
abordadas, no solo se constituyeron en fuentes para la investigación documental sino en dinamizadores de los contenidos producidos propiciando, en muchos casos, actividades en territorio que prolongaron el ciclo de vida de la narración concebida inicialmente como online.

El crecimiento que significó de Calles Perdidas marcó la apuesta de $\mathrm{Mu}$ jeres en venta: pensar un documental interactivo articulado con una serie de medios online y offline que pudieran ampliar el universo narrativo, llegar a diversos públicos en sus contextos de consumo y generar llamados a la acción para temas específicos. Este proyecto incluyó, así, una campaña gráfica con desarrollo de realidad aumentada, vídeos para LED ubicados en interiores y vía pública, una serie de cómics impresos y digitales, un documental para televisión emitido por una señal local, episodios para dispositivos móviles, un mapa interactivo y un libro.

\section{Conclusión}

En el marco de la comunicación digital, con aproximaciones a un lenguaje transmedia, el documental interactivo gana presencia en la región iberoamericana, frente al protagonismo de los principales países productores - Francia, Estados Unidos y Canadá -. Esta forma de representación de la realidad no se limita a plantear un visionado, sino que a través de los recursos del medio interactivo articula acciones sobre el mundo real, y viceversa. La conexión entre la representación virtual y la realidad física es uno de los pilares que posibilita el cambio, un vínculo entre la obra documental y la acción sobre el terreno, como evidencia el análisis de los casos estudiados.

Por otra parte, la temática social y el ámbito local como escenario redundan en la oportunidad de comunicar desde la proximidad, trasladando así realidades, necesidades, injusticias o conflictos. Constituye un compromiso con el entorno cercano y se propone, a su vez, un público universal al que informar, concienciar o interpelar, abandonando lo local hacia lo global.

El documental interactivo en Iberoamérica está reforzando su función de motor de cambio social: transformaciones de diferente escala que han llevado a denunciar, a la unión de la ciudadanía, a llevar a la actualidad aquello que se perdía en el olvido. A su vez, ha funcionado como elemento vertebrador de estrategias transmedia en las que, además de representar una realidad, trataba de aportar un valor añadido y dejar huella.

El futuro semeja prometedor, atendiendo a la consolidación de productores, instituciones, vías de financiación y proyectos de éxito. Al margen de la rentabilidad económica, la muestra analizada deja entrever, en este momento, un modelo especialmente comprometido con lo social y, por consecuencia, re- 
levante y necesario. El documental interactivo encuentra un hábitat apropiado en los espacios alternativos de creación, donde lo próximo se torna protagonista y demanda un cambio social.

\section{Agradecimientos}

Este artículo está elaborado en el marco del proyecto "Usos y preferencias informativas en el nuevo mapa de medios en España: modelos de periodismo para dispositivos móviles" (Referencia: CSO2015-64662-C4-4-R), del Ministerio de Economía y Competitividad (Gobierno de España) y cofinanciado por el fondo estructural FEDER, así como forma parte del programa de actividades de la Red XESCOM (Referencia: ED341D R2016/019) de la Consellería de Cultura, Educación e Ordenación Universitaria de la Xunta de Galicia.

El autor Jorge Vázquez-Herrero es beneficiario del programa de Formación del Profesorado Universitario financiado por el Ministerio de Educación, Cultura y Deporte (Gobierno de España).

\section{Referencias bibliográficas}

Aston, J. \& Gaudenzi, S. (2012). Interactive documentary: setting the field. Studies in Documentary Film, 6 (2): 125-139.

Aston, J.; Gaudenzi, S. \& Rose, M. (eds.) (2017). i-Docs: the evolving practices of interactive documentary. New York: Columbia University Press.

Calatayud, E. (2014). El webdoc como nuevo espacio de participación ciudadana: el caso de 0 Responsables (Barret Films 2013-2014). Dígitos, 1: 211-222.

Carpentier, N. (2015). Differentiating between access, interaction and participation. Conjunctions, 2 (2): 7-28.

Cortés-Selva, L. \& Pérez-Escolar, M. (2016). Civic participation and interactive documentaries: a contribution to the open government model. $E l$ profesional de la información, 25 (4): 578-587.

Domínguez, E. (2013). Periodismo inmersivo: fundamentos para una forma periodística basada en la interfaz y la acción. Tesis doctoral, Universitat Ramon Llull, Barcelona, España.

Favero, P. (2013). Getting our hands dirty (again): interactive documentaries and the meaning of images in the digital age. Journal of Material Culture, 18 (3): 259-277. 
Gantier, S. \& Labour. M. (2015). User empowerment and the I-Doc model user. In D. Bihanic (ed.), Empowering users through design: interdisciplinary studies \& combined approach for technological products and services. Cham, Switzerland: Springer.

Gaudenzi, S. (2013). The living documentary: from representing reality to cocreating reality in digital interactive documentary. Tesis doctoral, University of Goldsmiths, Centre for Cultural Studies (CCS), Londres, Reino Unido.

Gifreu, A. (2013). El documental interactivo como nuevo género audiovisual. Estudio de la aparición del nuevo género, aproximación a su definición y propuesta de taxonomía y de modelo de análisis a efectos de evaluación, diseño y producción. Tesis doctoral, Universitat Pompeu Fabra, Barcelona, España.

Gifreu-Castells, A. (2017). Interactive documentary aquí y ahora - here \& now: themes and directions in South America. In J. Aston, S. Gaudenzi \& M. Rose (eds.), i-Docs: the evolving practices of interactive documentary. New York: Columbia University Press.

Irigaray, F. (2015a). Periodismo transmedia: nuevas posibilidades narrativas y de experiencia de usuario. In G. Roitberg \& F. Piccato (coords.). Periodismo disruptivo. Dilemas y estrategias para la innovación. Buenos Aires: La Crujía Ediciones.

Irigaray, F. (2015b). Navegación territorial: entramado narrativo urbano. In F. Irigaray \& A. Lovato (eds.), Producciones transmedia de no ficción. Análisis, experiencias y tecnologías. Rosario: UNR Editora.

Jenkins, H. (2006). Convergence culture: where old and new media collide. New York: New York University Press.

Miles, A. (2014). Materialism and interactive documentary: sketch notes. Studies in Documentary Film, 8 (3): 205-220.

Nash, K. (2012). Modes of interactivity: analysing the webdoc. Media, Culture \& Society, 34 (2): 195-210.

Nash, K. (2014). Strategies of interaction, questions of meaning: an audience study of the NFBs Bear 71. Studies in Documentary Film, 8 (3): 221-234.

Nash, K.; Hight, C. \& Summerhayes, C. (2014). New documentary ecologies. Emerging platforms, practices and discourses. London: Palgrave Macmillan.

Ortuño, P. \& Villaplana, V. (2017). Activismo transmedia. Narrativas de participación para el cambio social. Obra Digital, 12: 123-144. 
Renó, D. P. (2013). Diversidade de modelos narrativos para documentários transmídia. Doc On-line, 14: 93-112.

Rose, M. (2017). Not media about, but media with: co-creation for activism. In J. Aston, S. Gaudenzi \& M. Rose (eds.), i-Docs: the evolving practices of interactive documentary. New York: Columbia University Press.

Vázquez-Herrero, J.; Negreira-Rey, M.-C. \& Pereira-Fariña, X. (2017). Contribuciones del documental interactivo a la renovación de las narrativas periodísticas: realidades y desafíos. Revista Latina de Comunicación Social, 72: 397-414.

\section{Documentales interactivos}

0 responsables (2013), de Barret Films y Asociación de víctimas del metro del 3 de julio, España. Disponible en: www.Oresponsables.com.

El charco azul (2013), de Ojo de Pez y Señal Colombia, Colombia. Disponible en: www.elcharcoazul.co.

Calles perdidas (2013), de Dirección de Comunicación Multimedial, Universidad Nacional de Rosario, Argentina. Disponible en: www.documedia.co m.ar/callesperdidas.

Chaco-PY (2014), de Andrea Ruffini, Paraguay. Disponible en: http://chacopy.com.

Illados (2014), de Laura Santos, Sibila Souto y Jorge Vázquez, España. Disponible en: http://illados.com.

4 ríos (2014), de Orgánica Digital, Colombia. Disponible en: http://4rios.co.

Mujeres en venta (2015), de Dirección de Comunicación Multimedial, Universidad Nacional de Rosario, Argentina. Disponible en: www.documedia.c om.ar/mujeres.

Proyecto Quipu (2015), de Chaka Studio, Chile, Reino Unido, Perú. Disponible en: https://interactive.quipu-project.com.

Pregoneros de Medellín (2015), de Grupo Creativo Carabalí y Thibault Durand, Colombia. Disponible en: www.pregonerosdemedellin.com.

Canción de la Ciudad (2015), de Facultad Libre, Argentina. Disponible en: http://cancionciudad.com.

Desiguales (2016), de Cátedra Latinoamericana de Narrativas Transmedia, Argentina. Disponible en: http://des-iguales.com.

El feriante (2017), de Facultad Libre y La Bemba del Sur, Argentina. Disponible en: http://elferiante.com.ar. 